\title{
Computing Boards for Internet of Things: A Comparative Survey
}

This paper was downloaded from TechRxiv (https://www.techrxiv.org).

\section{LICENSE}

CC BY 4.0

SUBMISSION DATE / POSTED DATE

$17-01-2022$ / 20-01-2022

CITATION

Datta Dhruva, Anantha; Babu, Sarath; Chakraborty, Abhishek; B. S., Manoj (2022): Computing Boards for Internet of Things: A Comparative Survey. TechRxiv. Preprint. https://doi.org/10.36227/techrxiv.18517235.v1

$\mathrm{DOI}$

10.36227/techrxiv.18517235.v1 


\title{
Computing Boards for Internet of Things: A Comparative Survey
}

\author{
Anantha Datta Dhruva, Student Member, IEEE, Sarath Babu, Member, IEEE, \\ Abhishek Chakraborty, Member, IEEE, and B. S. Manoj, Senior Member, IEEE
}

\begin{abstract}
The performance of present-day Internet of Things (IoT) computing boards is primarily based on their architectural designs and selection of appropriate application scenarios. This paper conducts a comprehensive and comparative survey of 60 popular and commercially available IoT computing boards with respect to various performance metrics. The metrics include the capability of processor, available interfaces, available modules for communication, available memory interfaces, power system-related specifications, over-the-air programming capability, Geo-location capability, shielding capability, compatibility with other IoT computing boards, and availability of community support/integrated development environment. Further, we highlight the selected 60 IoT computing boards' architectural advancements that result in diverse real-world application capabilities. Moreover, we also discuss possible application scenarios of the IoT computing boards and their implementation details in the context of selected performance metrics. Our comparative survey on the selected 60 IoT boards can be helpful for the IoT board manufacturers and the system developers to understand the stateof-the-art designs requirements. Furthermore, the insights can be used to develop IoT computing boards for appropriate future application scenarios.
\end{abstract}

Index Terms - Internet of Things, IoT, computing boards, architecture, performance analysis.

\section{INTRODUCTION}

$\mathbf{T}$ HE introduction of the Internet of Things (IoT) has transformed the way we interact with the world, from smart watches that monitor our activities and inform us about our health status to systems such as pacemakers which keep weak human hearts functional. In particular, present-day wireless/wired interactions around us have been part of the systematic progress of the Internet's evolution. The necessity and possibility of such interactions are primarily initiated by multiple active embedded systems that rely on the Internet for certain functions and reach the intended recipients. Therefore, to understand and compare the existing technological devices built on such wide platforms, it is necessary to understand IoT and its functionality.

There is no unique IoT definition and the introspection changes on the basis of application scenarios. For example, Radio Frequency Identification (RFID) tagging is one of the earliest implementations of IoT devices. Note, RFID tagging was invented during the World War II to identify enemy aircraft. The German military incorporated RFID-based

A. D. Dhruva, S. Babu, and B. S. Manoj are with the Indian Institute of Space Science and Technology, Thiruvananthapuram, Kerala 695547, India. A. Chakraborty is with the University of California, San Diego, CA 92093, USA. E-mail: \{ananthadattad, sarath.babu.2014, abhishek2003slg, bsmanoj\}@ieee.org. detection of spy planes in the vast landing regions of the mainland [1]. Also, object tagging with back-scattering was another early-days implementation for the RF-based passive device identification mechanism.

There exist different ways of looking into the definition of IoT. It can be explained based on the interactions of different intelligent devices (or things) which communicate among themselves by exchanging data and controlling information over wired or wireless medium. To begin, we mention the definition provided by the Institute of Electrical and Electronics Engineers (IEEE) [1]: "A network of items each embedded with sensors - which are connected to the Internet." Therefore, IoT can be considered as a network of uniquely identifiable items or things connected to the Internet. In addition, the IoT devices have unique sensing/actuation and potential programming capabilities, and the state of an IoT device can be updated/changed from anywhere at anytime, using the Internet connectivity of the device.

The concept of IoT has found its niche in many industries, such as manufacturing, environmental monitoring, transportation, medical, infrastructure management, home automation, energy management, media and entertainment, agriculture, and security [2]. Here, the application requirements can be achieved using specific devices, i.e., sensors, actuators, gadgets, appliances, or machines that can interact over the Internet using a wired or wireless connectivity. However, such devices are generally specific to their constraints and perks, thereby correlating with their functionalities.

However, one of the critical limitations of IoT devices is limited energy resources, especially in battery-driven IoT devices. In order to overcome energy storage-related limitations - upgrades in power storage, delivery, and acquisition systems are needed. Another limitation is the range of communication (i.e., transmission or reception) with which the IoT devices are operated. Note, communication range and data rate can be enhanced by improving RF efficiency with betterdesigned links or switching options to other types of wireless communication technologies. One such example is $\mathrm{Li}$-Fi which offers a very high communication link bandwidth [3]. Further, the need for better computation and storage capabilities are gradually increasing in today's IoT systems to perform computationally intensive tasks such as image processing or implementing large-sized machine learning- or deep neural networks-based operations [4].

A significant concern for any IoT system design is assuring the system security. Ill-conceived IoT systems are vulnerable to numerous cyber threats and frequently create severe 
damage to the systems. Such vulnerable IoT systems can be extremely harmful when real-world data processing and related applications are considered [5], [6]. Moreover, the application universality of the IoT systems is not achieved yet. As a result that, an IoT device designed for a specific set of tasks may have to be significantly modified to execute a different set of operations [6]. Therefore, the universality of IoT systems can also be considered a limitation.

Presently, there exist a variety of IoT computing boards ${ }^{1}$ This survey paper focuses on the 60 IoT computing boards and performs a comparative architectural study. We provide a complete list of 60 IoT computing boards as a supplementary document (refer to Annexure - I with the IEEE DataPort DOI: $10.21227 / j f 6 q-k 239$ ). In particular, this survey focuses on studying the IoT system implementation in conjunction with the world of embedded systems. Keeping in mind the previously discussed definition of IoT, we discuss the application possibilities and perform the comparative parametrization of the existing single board computing platforms. To the best of our knowledge, the survey of this IoT computing boards is one of its first kind.

Rest of the survey is organized as follows: In Section II the motivation behind the comparative survey of the IoT computing boards is highlighted. The performance metrics for comparing all the 60 IoT boards are described in Section III. Section IV, on the other hand, presents a comparative study among the chosen IoT board. Next, in Section V, key observations on the selected IoT computing boards are summarized. At last, Section VI concludes the survey with a few future research directions.

\section{Motivation}

The evolution of the embedded systems, which is one of the cornerstones for the development of the present-day IoT computing boards, become possible due to the advancements in the domains of embedded computing, miniaturization, growth of the Internet, integrated development tools, and software accessibilities [7]. The embedded systems-based devices have incorporated in such a way that there exist very few electrical devices that do not have inbuilt embedded systems. This advancement is possible due to the unique ability of microprocessor and microcontroller industries to simplify many approaches such as better handling of product range, better handling of user applications, maintenance of existing products, and the growth in the technology domains. Nowadays, even college undergraduates often use embedded system boards, such as Arduino and Raspberry $\mathrm{Pi}$, that are readily available, cheap, and supported by open-source operating systems and application software along with a rich community support.

It can also be observed that the Internet has been evolving rapidly and changes our daily lives in terms of digital connectivities and the application domains. Further, presentday embedded systems incorporate IoT principles to enhance application domains and user experience and fulfill various

\footnotetext{
${ }^{1}$ Here, "IoT computing boards" and "IoT boards" are synonymous, and we use them interchangeably in this paper.
}

List of Acronyms

\begin{tabular}{ll}
\hline ADC & Analog-to-Digital Converter \\
AP & Access Point \\
ATA & Advanced Technology Attachment \\
BLE & Bluetooth Low Energy \\
CAN & Controller Area Network \\
DAC & digital-to-Analog Converter \\
DDR & Double Data Rate \\
DRAM & Dynamic Random Access Memory \\
ECU & Electronic Control Unit \\
EEPROM & Electrically Erasable Programmable RAM \\
GNSS & Global Navigation Satellite System \\
GPIO & General Purpose I/O \\
GPRS & General Packet Radio Services \\
GSM & Global System for Mobile Communications \\
I2C & Inter-Integrated Circuit \\
I/O & Input/Output \\
IEEE & Institute of Electrical and Electronics Engineers \\
IoT & Internet of Things \\
ISA & Industrial Standard Architecture \\
ISM & Industrial, Scientific, and Medical \\
LAN & Local Area Network \\
LoRa & Long Range \\
LTE & Long-Term Evolution \\
NB-IoT & Narrow Band IoT \\
PCI & Peripheral Component Interconnect \\
RAM & Read-Only Memory \\
RFID & Radio Frequency Identification \\
RISC & Reduced Instruction Set Computer \\
SARM & Serial Advanced RISC Machine \\
SCSI & Small Computer System Interface \\
SDRAM & Synchronous DRAM \\
SoftAP & Software AP \\
SPI & Serial Peripheral Interface \\
SRAM & Static RAM \\
UART & Universal Asynchronous Receiver/Transmitter \\
\hline & Universal Serial Bus \\
USB &
\end{tabular}

industries' requirements. Therefore, when a new industry aims to implement IoT-based system design, it must perform extensive requirement analysis and foresee available advancements in existing systems. Similarly, detailed and comparative knowledge about the existing technological innovations on the research front is required to incorporate the next-level of advancements. Our observations, which led to this comparative survey, summarize the state-of-the-art advancements of the selected 60 IoT computing boards and also highlight future IoT computing board development and prototyping directions.

Note, it is essential to quantify a few specific properties of the selected IoT boards to enable system designers and researchers to select appropriate IoT boards for their system designs/experiments. In this survey, we attempt to document a unique understanding of how the existing IoT boards cater to the needs for rapid prototyping and development by quantitatively comparing a few critical performance metrics. In order to understand this evolving enhancement, we utilize a handful important performance metrics for the classification of the selected IoT boards and perform a comparative analysis to gain insights about the developments of the future IoT boards 
in a holistic approach.

\section{Performance Metrics for Classification}

We select the following performance metrics to represent a set of standard features for the selected 60 IoT boards: (i) processor capability, (ii) available interfaces (serial and parallel), (iii) communication modules available, (iv) memory interface, (v) power system-related specifications, (vi) overthe-air programming, (vii) Geo-location capability, (viii) availability of shields, (ix) compatibility with other IoT boards, and (x) community support/integrated development environment. In the following, each of the performance metrics is discussed briefly.

\section{A. Processor Capability}

Every embedded system design includes a microcontroller or a microprocessor to execute commands and process certain information, handle tasks autonomously, process or relay data, and perform the IoT system's core functionalities. Note, processor capabilities can be defined based on the following parameters:

(i) Clock speed gives an approximate measure of the number of instructions executed per second.

(ii) Graphic acceleration specifications deal with highquality videos and photos.

(iii) The number of cores helps execute parallel computation.

Therefore, the selection of a particular processor is primarily based on the specifications of each of the previouslymentioned parameters. Moreover, many logical and computational operations can be implemented over the processors when specific IoT applications are considered.

\section{B. Available Interfaces}

Interfaces that communicate with sensors, actuators, and other machines are essential components in an embedded system. Such interfaces are generally made according to specific standards for commonality of implementation and expansion. Interfaces can widely be classified into two categories: serial and parallel interfaces. Note that the two interfaces are generally compared based on the type, speed, and quantity of the specific interface type in a system. Each interface also consists of an I/O port for communication to the external world. In the following, each of the interfaces is highlighted.

a) Serial Interface: Numerous serial interface options are being implemented with varying data rates, the complexity of implementation, and the number of possible devices connected to a particular interface. For each embedded IoT system, a serial interface mechanism is required to interact with other devices within the system. There can be actuators, sensors, or machines that send and receive data in a particular format (most widely a serial interface). Among the available serial interfaces, the following serial interfaces are chosen to perform a comparative analysis:

(i) Controller Area Network (CAN) is one of the most widely used protocols in the automobile industry and handles many crucial aspects of an automobile system without a host system. In particular, CAN protocol is similar to the nervous system of automobiles and interconnects Electronic Control Units (ECUs) to form a network. A generated message at a node (i.e., an ECU) is broadcast by CAN to other nodes in the network, and, based on the relevance of the message, a particular action is taken at a selected node. Note that each message has its priority and can deliver the generated messages based on their importance. Therefore, CAN is a message-based protocol and is very reliable, efficient, simple, single bus, and fully centralized [8].

(ii) Ethernet is a family of wired Internet protocols used widely to access the Internet. An Ethernet cable has a physical encased wiring over which data packets are transmitted and helps users to interact with Local Area Networks (LANs) or Wide Area Networks (WANs). Note, Ethernet infrastructure is backward compatible, economical, and also able to provide speed up to 400 Gbps [9].

(iii) Serial Peripheral Interface (SPI) is a synchronized data transmission system with master-slave configuration and capable of simultaneously sending and receiving data packets, i.e., with the help of a full-duplex mechanism. SPI is a 4-pin wired interface with multiple architectures available for use, and an example is multiple slaves and a Daisy chain. Note, this approach is widely used in embedded systems while interconnecting electronic components such as Analog-to-Digital Converter (ADC), Digital-to-Analog Converter (DAC), shift register, and Serial Advanced RISC Machine (SARM) [9], [10].

(iv) Inter-Integrated Circuits (I2C) is an asynchronous data exchange system that utilizes two wired connections with one wire carrying a clock and the other transporting data. I2C is most widely used for short-distance communication between controller chips and other subsystems in a device. Different modes of operation of I2C makes it convenient for implementing application-specific frequency rate to transfer data packets [10].

(v) Universal Asynchronous Receiver/Transmitter (UART) is one of the oldest serial communication protocols used for connecting teletypes to minicomputers. In particular, UART is a straightforward messaging system with a start bit and an end bit tagged along with a parity bit for data communication. Note, UART is a 2-wired protocol with the following modules: (i) transmitter and (ii) receiver. A full-duplex system can be implemented using UART protocol with a data transfer rate up to 115,200 baud rate [9].

(vi) Universal Serial Bus (USB) is one of the most famous serial interfaces to connect a wide range of peripheral devices. Note that USB is designed with different form factors for multiple device sizes. USB is also used as a powering up of the devices [9]. There are various USB versions, with the most recent one is USB 3.0 and a data transfer rate of approximately $5 \mathrm{Gbps}[11]$.

b) Parallel Interface: Though parallel communication interfaces are rarely used in embedded IoT systems, we observe the availability of parallel interfaces such as Industrial 
Standard Architecture (ISA), parallel Advanced Technology Attachment (ATA), Small Computer System Interface (SCSI), Peripheral Component Interconnect (PCI) bus, and General Purpose Interface Bus (GPIB IEEE-488). The above technologies are seen in the pool of selected boards. Note, we include various parallel interfaces in our study.

\section{Communication Modules}

A communication module interacts with the processor and the network, thus influencing network processing requirements, application expansions, and required hardware drivers. There exist many diverse communication modules, such as different versions of Wi-Fi, Bluetooth, and Ethernet. The following are the critical aspects of the communication modules:

(i) The first key aspect of the communication module is the communication protocol in use and the corresponding standard for the IoT computing boards. There are numerous standards such as Long Range WAN (LoRaWAN), Wi-Fi, Bluetooth Low Energy (BLE), and Optical links based on Li-Fi.

(ii) The next central avenue of the communication module is the flexibility of the protocol used. For example, more than one version of a particular communication protocol is mentioned earlier in this paper. Also, specific boards can perform flexible operations within the family of protocols. This parameter defines the compatibility parameter of the IoT boards.

(iii) Further, every wireless communication standard is possible only with an On-Chip/ Integrated antenna. That is, specific boards are replaceable based on application requirements.

An important aspect is the availability of wired and wireless communication interfaces to choose the boards specific to the application in some instances. Each of the chosen communication protocols is discussed below:

a) Wi-Fi and Wi-Fi/SoftAP: Wi-Fi belongs to the IEEE 802.11 standard family of wireless communication protocols. In this paper, we consider varied versions of the WiFi family of protocols such as IEEE 802.11a, IEEE 802.11b, IEEE $802.11 \mathrm{~g}$, and a combination of IEEE $802.11 \mathrm{a} / \mathrm{b} / \mathrm{g} / \mathrm{n}$. Each of such protocols deals with the properties of the Wi-Fi module and its capabilities. Wi-Fi provides a communication range of 20 meters for indoor and approximately 100 meters for outdoor scenario [12]. Software Access Point (SoftAP) is the capability of an IoT module to form an access point for network connectivity for Wi-Fi. The module hardware may not be originally designed as a router. However, a certain set of software can transform the IoT board with SoftAP into a virtual router. This capability is also studied for various boards in Section $\mathrm{V}$

b) Bluetooth: Bluetooth is a part of the IEEE 802.15.1 standard. It is a short range (typically about 8 meters), low cost, and low power consumption suitable for communication between close-range mobile devices. Furthermore, an ultra low power version of Bluetooth named BLE is also a potential communication mode for future IoT computing boards [12]. Although BLE offers lower data rates compared to Bluetooth, a significant number of sensor nodes and applications can fulfill this requirement [13].

c) LoRaWAN: Developed by the LoRa Alliance (open and non-profit association), LoRaWAN is a recent technology used for long-range communication, and it is specifically designed for IoT networks. Although LoRa has a low data rate compared to other protocols (typically $0.3 \mathrm{kbps}-50 \mathrm{kbps}$ ), it realizes an operational system of nearly 30 kilometers in range without the presence of obstacles and is also generally designed with a battery-operated lifetime of up to 10 years. LoRaWAN operates in the Industrial, Scientific, and Medical (ISM) band, and it aims to have a unified protocol that defines a global standard for various IoT operators [12].

d) Cellular Technology: Cellular networks operate over terrestrial areas, including 2G, 3G, 4G, and 5G networks. Each cell is equipped with a cellular transceiver that will establish a communication system within the subscribed mobile devices, and this can be utilized to build a very wide IoT network [14]. This paper compares some of the boards capable of networking with the cellular technologies using the Global System for Mobile communication (GSM) standards and Long Term Evolution (LTE) standards. IoT systems can potentially use such well-established cellular technology to meet application demands. The $2 \mathrm{G}, 3 \mathrm{G}$, and $4 \mathrm{G}$ networks are now the most prominent ones in the cellular industry, which often offer lowcost, high expansive networking systems with constrained data rates for wireless IoT. Specific boards under consideration have these capabilities.

e) SigFox: SigFox is a global network operator established in France that developed a technology for wireless networks for low-power devices configured for continuous data transmission at periodic time intervals. SigFox operates in the ISM band to provide network coverage to large land areas. SigFox is also capable of handling dense networks and is promising for IoT network expansions [15].

\section{Memory Interfaces}

The amount of memory supported can roughly represent the addressing capability of the processor. The memory interface also implies the memory card slot provision other than the device's internal memory and plays an essential role in handling the onboard processing of certain information. The memory capacity also determines the data transfer rate required in conjunction with the application the device is oriented to be used. This survey selects IoT boards with various memory storage options such as SRAM, DRAM, EEPROM, DDR2, DDR3, and Flash. A combination of different storage types can be found on each board. A brief explanation for each storage variant is mentioned in the following.

a) Static Random Access Memory (SRAM) and Dynamic Random Access Memory (DRAM): Random-access memory storage employs flip-flops to manage the storage of a bit. The static variety of RAM employs only latching circuitry to manage the storage, whereas the Dynamic RAM variety uses a refreshing circuit to maintain the bit information constantly. SRAM has a faster response time and has a low packing density compared to DRAM [16]. In the comparative study, 
we have visualized the contribution of these data storage in a particular board.

b) Electrically Erasable Programmable Read-Only Memory (EEPROM): A variety of read-only memory devices are generally included in IoT computing boards to store firmware essential for the board's operation. The storage size is generally tiny, in kilobytes or less. We rarely find EEPROM storage usage in modern boards. However, Arduino utilizes this storage system in most of its boards to store the operational firmware. EEPROM is used in RFID tags to enable reconfigurable RFID card set for various applications [17].

c) Double Data Rate (DDR) Synchronous Dynamic Random-Access Memory Series: DDR series is a descendant of the Synchronous SDRAM that utilizes a clock to enhance the memory transfer speed and capacity. There are various versions of DDR memory available: (i) DDR that uses double data rate architecture and enables the transfer of two data words in one clock period [18], (ii) DDR2 surpasses DDR by higher speed and low power requirement and executes process using pipe-lined, and multi-bank architecture [18], (iii) DDR3 is an updated production of DDR2 and provides a memory reset option with increased data transfer rate [19], and (iv) DDR4 has larger operational bandwidth, increased frequency, and lower power consumption to give better data rates [20].

Availability of options for cloud-based storage associated with the board is also a viable futuristic option for the currently available boards.

\section{E. Power System-related Specifications}

This parameter influences the duration of autonomous operation and overall performance metric in power consumption and operational ease. We classify the power-related specifications into two categories.

- Battery-powered systems

1) The capacity of the battery

2) Number of service hours before complete discharge

3) Lifetime and life cycle of the battery

- Wall socket/Adapter powered

1) Adapter specifications

The previously mentioned parameters form the basis of the analysis to classify the boards into various categories of power operations, and for a user, a particular board can be identified to best suit the application and compared accordingly.

\section{F. Over-the-Air Programming}

Over air programmable devices incorporate a mechanism in which software can be distributed between different nodes in an IoT network. The software is sent to the nodes as firmware updates and is executed automatically without the respective nodes' intervention or authorization. Similar to the firmware updates for enhancing security in smart phones [21], as the standards evolve for more security, this over-the-air programming is employed for distributing the software to enhance user experience and security [22]. The same technique is now being incorporated into upcoming IoT devices, where we can send new codes that are captured, stored, and utilized for further operations by the IoT nodes. This process can be facilitated over a networking protocol such as Wi-Fi, LoRa WAN, BLE, Cellular, and many other wireless or wired standards [23].

\section{G. Geo-location Capability}

Geo-location is an ability to identify the position of the device using specific satellite systems such as Navstar GPS [24], [25] and NAVIC [26]. Each of the satellite systems has an associated receiver mechanism. The information we obtain from them can help us identify our position, direction of motion, and velocity in near real-time. An example of it being used is IoT achieving its place in space satellite systems [27]. We need to consider this new Geo-location capability as a helping hand in many applications connected to navigation, guidance, and control.

\section{H. Shielding Capability}

Shields are commonly referred to as hardware or software components added to available boards/ modules. The shields are commercially available for use and are specific to certain applications [28], [29]. The additions may include interface extension, additional antenna, respective circuitry, or additional computational support for image processing. Such expansible capability for an IoT board can push the board to become a generic design applicable for many operations.

\section{Compatibility with Other Boards}

With many boards coming into the market, a new goal emerges in the developer community of being pin to pin compatible with other widely used boards such as Arduino. A commonality in pin layout and software support systems is essential as it allows the end-user to interoperate various boards with much more freedom. This principle makes addon components to these development boards, and the add-on hardware components are called shields. This compatibility in pin connections and software will improve the application range of particular hardware used in an IoT system.

\section{J. Community Support/Integrated Development Environments}

Community support refers to the amount of information and discussion forums available on specific boards usage from the users. Such forums play an essential role in the faster development of a product. Such forums also provide easier debugging as the community enthusiasts experiment and bring out innovative solutions and, in turn, help in developing the board in ways not perceived. A developer community is generally a voluntary setup run by individuals from all over the world that are active developers of many boards, trying to develop applications collaboratively. Integrated Development Environment (IDE) is a software module that helps us interact with the IoT computing board and simplify the process of deployment and operation. IDEs provide efficient mechanisms to configure source code, build automation tools, and help to debug. In addition, some IDEs provide syntax highlighting, 


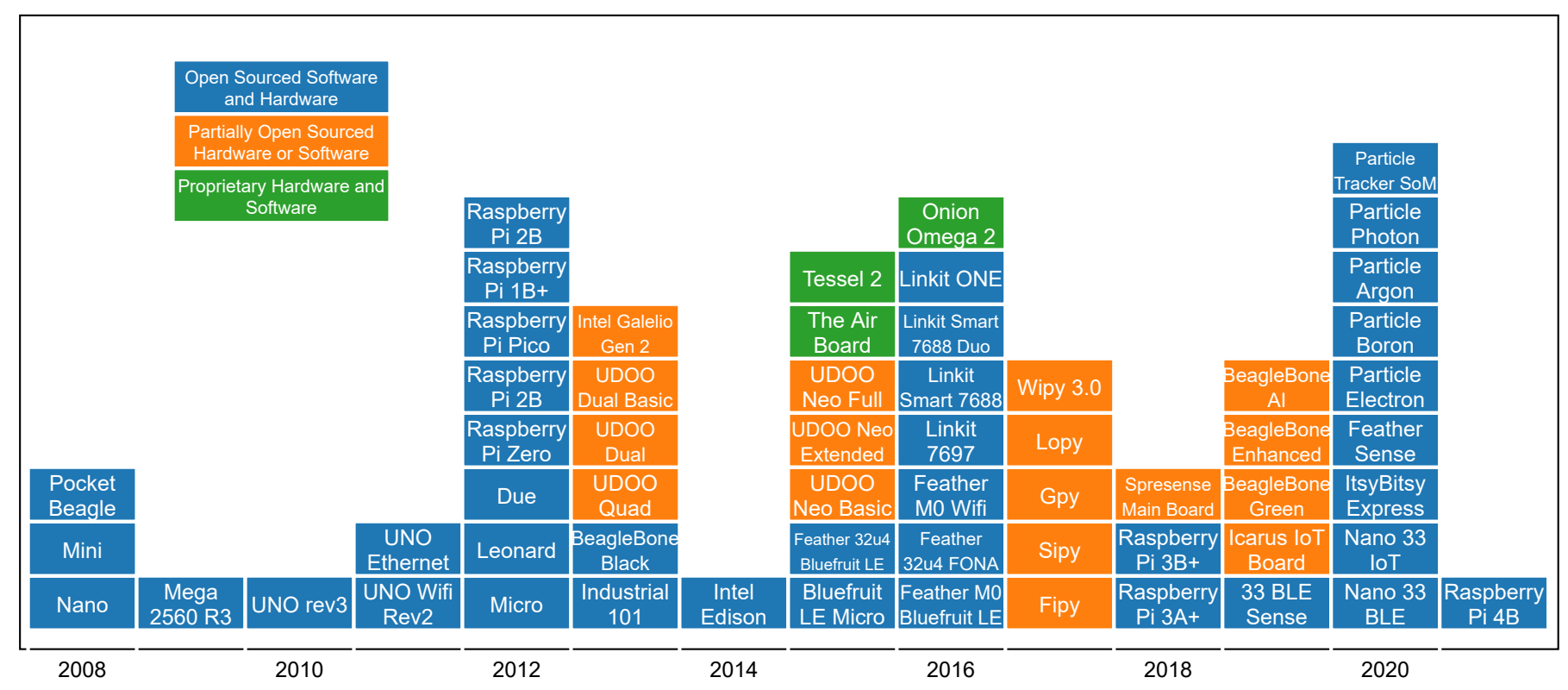

Fig. 1: The year-wise release of the IoT computing boards.

code completion, version control for software, code searching, and visual programming capability.

Fig. 11 shows us the year-wise release of the computing boards considered for the analysis; various shades show the level of open-source from a particular board. They give us an idea about the distribution of boards we refer to here. The level of open-source, as mentioned in the figure, is calibrated as follows:

1) Open Sourced Software and Hardware that are readily available on the Internet with complete support from the designer(s).

2) Partially Open-sourced Hardware or Software where some parts of an IoT board may have Intellectual Property (IP) protection. Any such board with an IP-protected segment can be considered in this category.

3) Proprietary Hardware and Software where the designers have complete rights over the hardware they use and the software they provide.

\section{Comparative ANALYsis OF THE IOT COMPUTING BOARDS}

The chosen IoT computing boards highlight the advancements in the embedded IoT domain by characterizing the architectural aspects and comparing them based on the metrics defined in Section IIII The metrics provide us numeric data that are analyzed in Section $\mathrm{V}$. Moreover, the extracted data can be used to characterize the boards' capabilities. The metrics under analysis are listed as follows:
A Interface provision
B Clock cluster
C Memory distribution
D IDE/Community support
E Internet interface provision
F Special provisions

\section{A. Interface Provision}

The interface analysis considers the number of available analog and digital pins which serve as a standard interface. The pins are performing actions corresponding to the protocols discussed in metrics of classification Section III-B

We considered the number (or, count) of analog and digital pins in each board to indicate the interface provisioning. There is also a statistical indicator for the number of boards with the same total number of pins in Fig. 2 .

The number of pins gives us the range of applications realizable by the specific board. Many boards tend towards rapid prototyping that necessitates identifying the number of pins for a particular application. The distribution of several pins will also make us aware of interface possibilities, and this distribution is indicated in Fig. 3 .

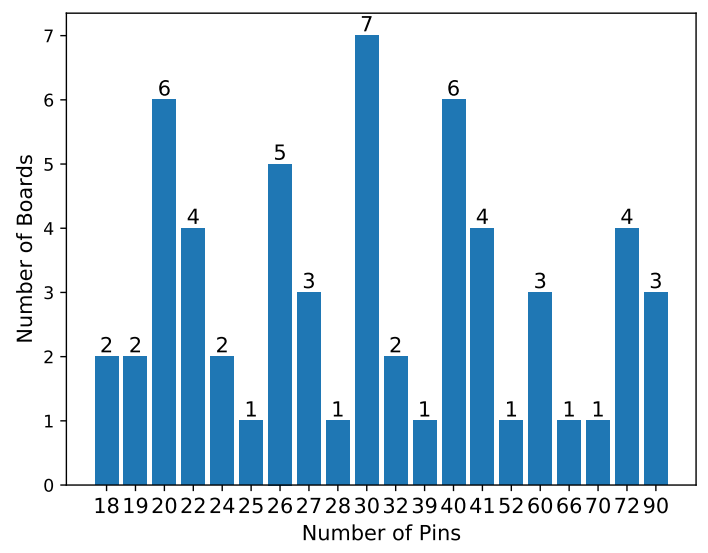

Fig. 2: Distribution of 60 IoT computing boards with the total pin numbers.

Each board has a unique architecture for handling the interfaces and, hence, to accommodate the application point 
of view, we list down the number of analog and digital format pins available on the board in Fig. 3. The analog pins include the ADC-enabled pins. The digital pins include every other pin, including the power provision pins, GPIOs, and also the protocol-specific pins for interaction in various interface formats mentioned in Section III-B.

The analog-to-digital pins ratio on the boards shows us a common trend of allocating a fixed number of analog pins (i.e., ADC). The trend has shown extensive use of digital interfaces that indicate the digitization of the sensor nodes (modules used are now read-out in digital) in IoT. Such digitization makes them more reliable with in-house instrumentation and sensing capabilities. A simple statistic is used to find the average analog-to-digital pin ratio and can be represented as follows:

$$
\text { Analog to Digital Ratio }=\frac{\text { Average Number of Analog Pins }}{\text { Average Number of Digital Pins }},
$$

where

$$
\text { Average Number of Analog Pins }=\frac{\sum_{i=1}^{N} \text { Analog Pins on Board }}{\text { Total Number of Boards }(N)}
$$

and

$$
\text { Average Number of Digital Pins }=\frac{\sum_{i=1}^{N} \text { Digital Pins on Board }}{\text { Total Number of Boards }(N)} \text {. }
$$

The rounded-off average number of analog and digital pins are 8 and 30, respectively, as the computing boards are considered. The ratio of analog-to-digital is measured at $24.46 \%$. The numbers indicate a significant average of nearly $25 \%$ of the pins being given for the analog sensing interface. The analog sensing requirement can be an effective sensor selection and rapid prototyping option.

\section{B. Clock Cluster}

The processor clock speed is directly related to the rate at which instructions can be executed on the board. Here, the clock speed determines computation performance and the data transfer rate through an interface. Therefore, analyzing the clock speed helps choose the board that satisfies the application requirement. Our analysis on the chosen IoT computing boards has shown that approximately $42 \%$ of the boards have a clock speed less than $100 \mathrm{MHz}$, indicating that all of the boards with a clock speed less than $100 \mathrm{MHz}$ suffice the requirements set by many IoT applications.

Fig. 4 shows that the clock cluster based on the clocking speeds used by the boards, i.e., the number of boards that have the same clock speed, is shown. In order to make a clustered analysis, we have classified the boards into three categories: (i) very high clock speed, (ii) high clock speed, and (iii) lower clock speed in a relative sense. We use a clustering method, with outliers set to $50 \%$, to get the lower half of the clock speeds corresponding to low clock speed boards and less than $192 \mathrm{MHz}$ clock speed. The other half is split into very high and high, respectively. This classification is based on $50 \%$ outliers, where the high clock speed corresponds to the boards with clock speed beyond $800 \mathrm{MHz}$ as shown in Table [1]

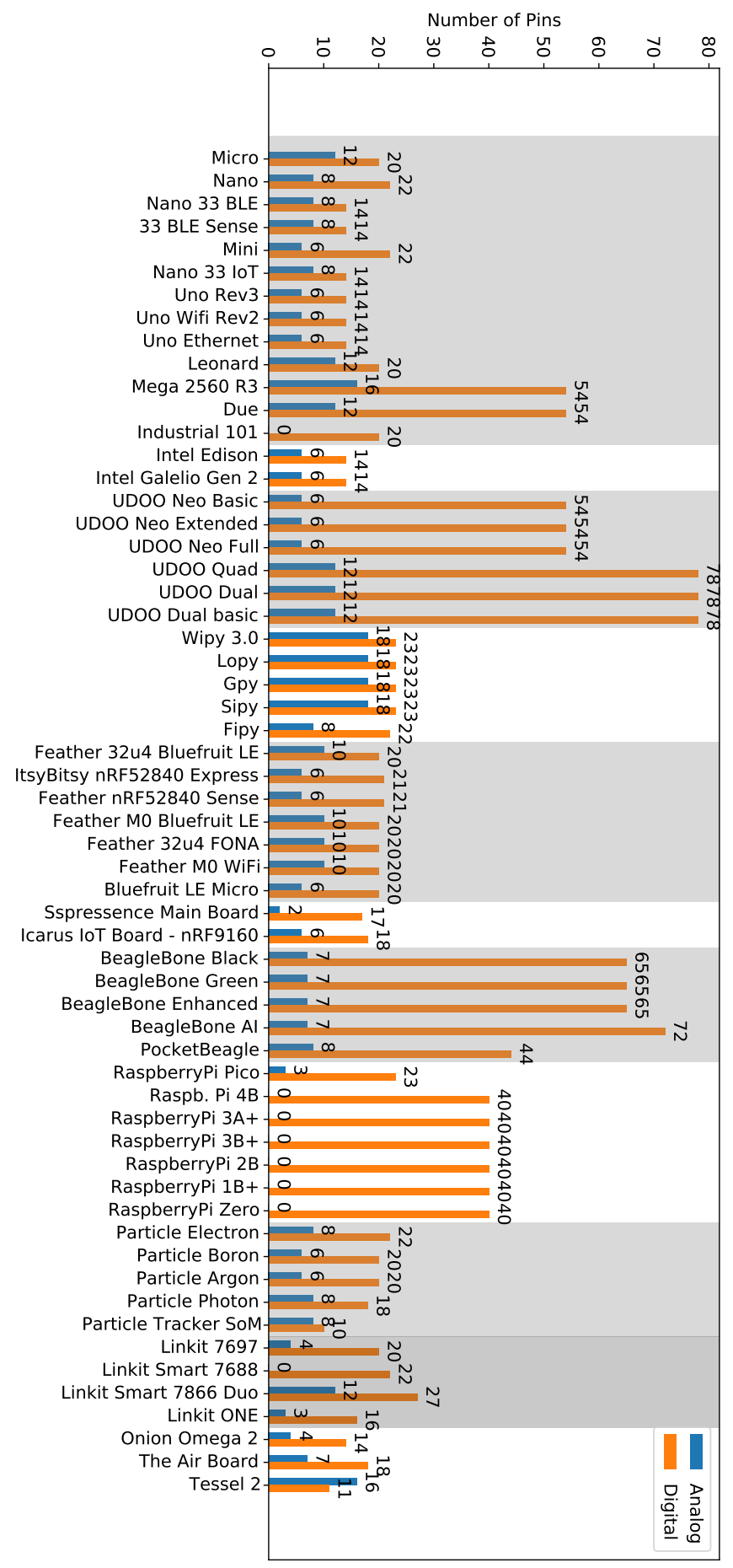

Fig. 3: Number of analog/digital pins available in each IoT computing board. Here, shading represents associations of the boards with their manufacturers.

The processing capability is also reflected by the specific computing board's number of cores. The multi-threading capability and multiprocessing capability are essential for industrial applications. An example is the Electronic Control Units (ECUs) used in the automotive industry, where the tasks of the vehicle are distributed between the cores to support the functioning of the node based on the critical quality [85]. Our analysis shows that 34 boards have a single-core processor, 20 boards are equipped with dual cores, another with three cores, 


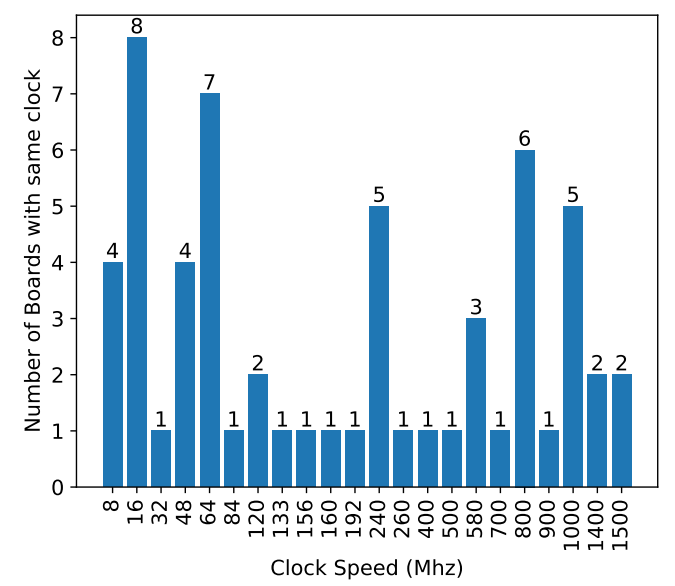

Fig. 4: The distribution of clock-speeds for the chosen 60 IoT computing boards.

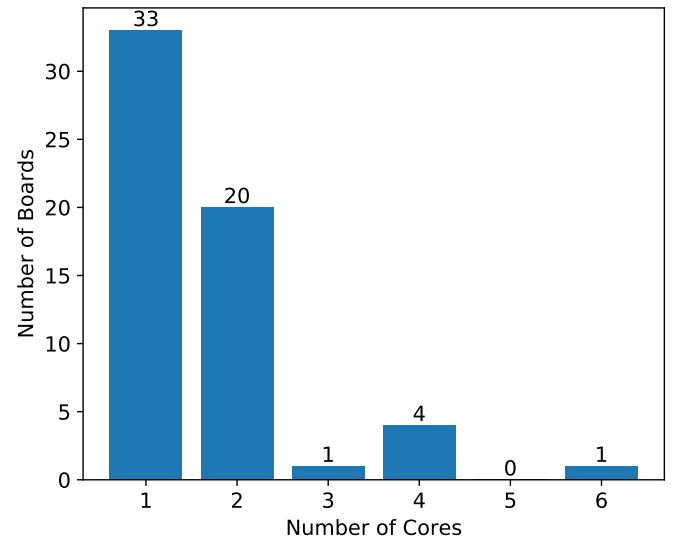

Fig. 5: Number of IoT computing boards with the same number of cores.

five boards with quad-core, and another with six cores, as can be seen from Fig. 5 .

\section{Memory Distribution}

The memory distribution is analyzed based on the proportion of each memory segment provided to the board. The boards we have analyzed are associated with different memory types, including EEPROM, SRAM, DRAM, Flash, DDR2, and DDR3 based memory segments. In addition, there exist boards associated with SD card slots that can support extended memory interface provision.

The diversity of memory types is also associated with their usage trend and similarly correlated with their application domains. An example of such instance is Arduino adopting EEPROM to store its bootup where IDE interface management modules are hereditary and can be seen in many boards of Arduino. Conversely, DDR2 and DDR3 storage have been widely adopted by IoT boards as the computational requirement and storage requirements are increasing.

Fig. 6 shows the year-wise provision of various types of memory segments, summed up for all the chosen boards. We notice some peculiar trends, such as increased SRAM and Flash memory used in the devices (and replaced EEPROM) as they progressed from 2008 to 2020 . A Similar trend of the
TABLE I

Clustered Table of Computational Capability

\begin{tabular}{|c|c|}
\hline Speed of Computation & Name of the Boards \\
\hline \multirow{12}{*}{ Very high clock-speed boards } & RaspberryPi 4B $\mid 30$ \\
\hline & BeagleBone AI $|31|$ \\
\hline & RaspberryPi 3A+ 32 \\
\hline & RaspberryPi 3B+ 33 \\
\hline & BeagleBone Black |34] \\
\hline & BeagleBone Green 35 \\
\hline & BeagleBone Enhanced 36 \\
\hline & PocketBeagle 37 \\
\hline & Raspberry Pi Zero [38] \\
\hline & Raspberry Pi 2B 39 \\
\hline & UDOO Neo Series 40 \\
\hline & UDOO Dual and Quad Series 41 \\
\hline \multirow{18}{*}{ High clock-speed boards } & Raspberry Pi 1B+ $|\overline{42}|$ \\
\hline & Onion Omega $2[43]$ \\
\hline & Linkit Smart 7688 44 \\
\hline & Linkit Smart 7688 Duo | 44 \\
\hline & Intel Edison [45], |46] \\
\hline & Arduino Industrial 101 [47] \\
\hline & Linkit ONE [48] \\
\hline & Pycom Fipy $\overline{\overline{49}}$ \\
\hline & Pycom Wipy4.0 [50] \\
\hline & Pycom Lopy \\
\hline & Pycom Gpy [52] \\
\hline & Pycom Sipy \\
\hline & Linkit 7697 |54] \\
\hline & Icarus IoT Board - nRF9160 [55 \\
\hline & Spresense Main Board [56] \\
\hline & Raspberry Pi Pico [57| \\
\hline & Particle Photon 58 \\
\hline & Particle Electron |59| \\
\hline \multirow{26}{*}{ Low clock-speed boards } & Arduino Due $[\overline{60}]$ \\
\hline & Particle Boron 61 \\
\hline & Particle Argon $|\overline{62}|$ \\
\hline & Particle Tracker SoM 63 \\
\hline & ItsyBitsy nRF52840 Express 64 \\
\hline & Feather nRF52840 Sense 65 \\
\hline & Nano 33 BLE [66 \\
\hline & 33 BLE Sense 67 \\
\hline & Nano 33 IoT $|68|$ \\
\hline & Feather M0 Bluefruit LE [69| \\
\hline & Feather M0 Wi-Fi $|70|$ \\
\hline & Tessel 2 [71] \\
\hline & Intel Galileo Gen 2 \\
\hline & Micro [73] \\
\hline & Nano 74 \\
\hline & Mini $|75|$ \\
\hline & Nano 33 IoT 68 \\
\hline & Uno Rev3 $\mid 76$ \\
\hline & Uno Wi-Fi Rev2 |77| \\
\hline & Uno Ethernet \\
\hline & Leonard $\mid 79$ \\
\hline & Mega 2560 R3 [80] \\
\hline & Feather 32u4 Bluefruit LE 81 \\
\hline & Feather 32u4 FONA 82 \\
\hline & Bluefruit LE Micro 83 \\
\hline & The Air Board 84 \\
\hline
\end{tabular}

increased use of DDR2 and DDR3 memory segments can also be seen.

\section{IDE/Community Support}

Each board is supplemented with a specific combination of storage types and can be visualized with two different sets 


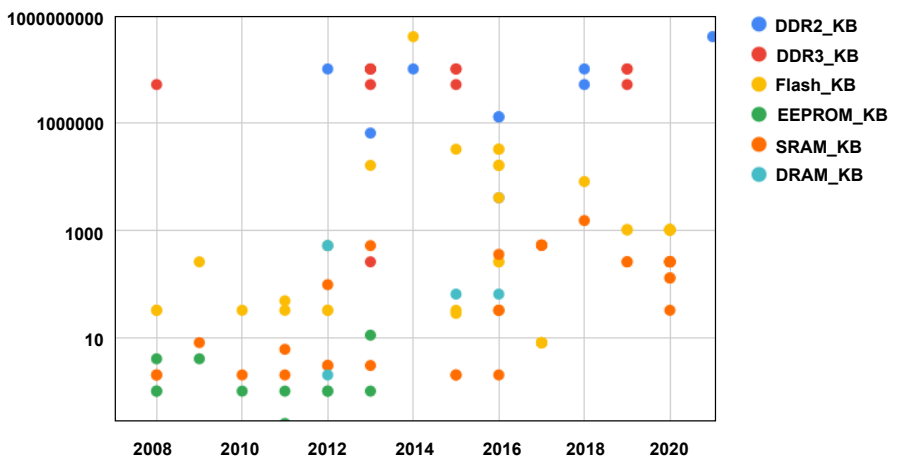

Fig. 6: Year-wise measure of various memory sizes allocated from each of the chosen IoT computing boards.

of graphs. In particular, memory distribution using DRAM, SRAM, and EEPROM is shown in Fig. 7, and memory allocations with DDR2, DDR3, and Flash is depicted in Fig. 8,

It can also be noted that the most recent compact storage versions of the SD cards are also available for a few IoT boards. In addition, the SD cards have OS support for faster prototyping along with memory extension capabilities. Out of the considered 60 IoT boards in our survey, 21 IoT boards comprise SD card support.

In the following, we provide information about the available IDE packages and the qualitative expanse of the community of users for a chosen board. We segmented the boards into multiple categories based on their vendors: Arduino, Raspberry $\mathrm{Pi}$, Adafruit Industries, MediaTek, Onion Company, Pycom, BeagleBone, Particle, Linkit, and Other sets of boards. The analysis done has all data acquired from the vendor-provided datasheets.

1) Arduino: Arduino has been one of the major players in the rapid prototyping and IoT domain. In particular, the architecture and community support always give an edge. The IDE also proves to be an essential aspect of these boards. It is an open-source project that makes both the boards' hardware and software developed by the open research community. The boards considered from the Arduino family are as follows:

(i) Uno, (ii) Micro, (iii) Nano, (iv) Mini, (v) Due, (vi) Leonard, (vii) Mega, (viii) M0, (ix) Yun Mini, (x) Uno Ethernet, (xi) Tian, and (xii) Mega ADK.

2) Raspberry Pi: Like the Arduino community, the Raspberry $\mathrm{Pi}$ family dominates generic computing and highercapacity open-source boards. Besides the community support, the software is extensive due to its generic compatibility. The boards considered from the Raspberry Pi family are as follows:

(i) Raspberry Pi Nano, (ii) Raspberry Pi 4, (iii) Raspberry Pi 3 B+, (iv) Raspberry Pi Zero, and (v) Raspberry Pi 2.

3) Adafruit Learning Systems: Like Arduino, Adafruit Learning Systems is also a hardware and software opensource project with a huge catalog of boards and their respective shields. Also, providing learning tutorials and community support is comparable to the Arduino community. The boards considered from the Adafruit family are as follows:

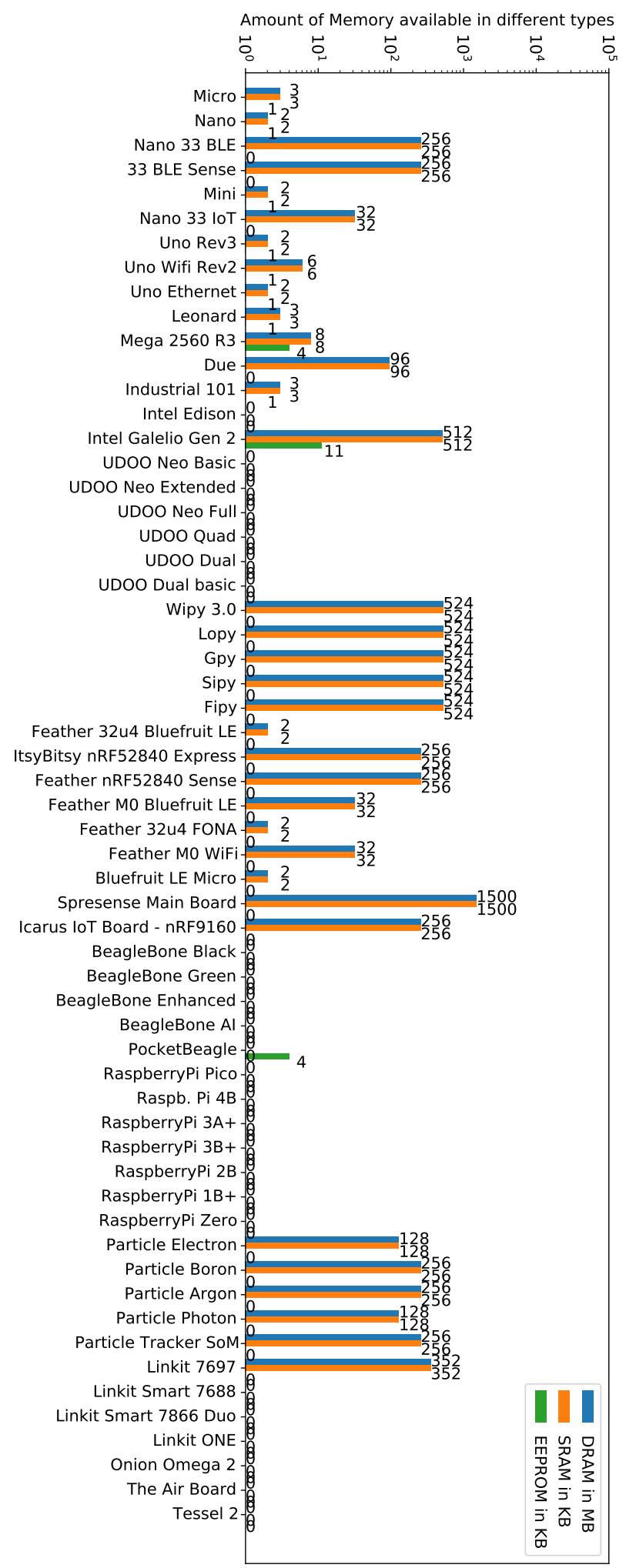

Fig. 7: Distribution of memory of each of the IoT computing boards for a combination of DRAM, SRAM, and EEPROM memories.

(i) Feather 32u4 Bluefruit LE, (ii) ItsyBitsy nRF52840 Express, (iii) Feather nRF52840 Sense, (iv) Feather M0 Bluefruit LE, (v) Feather 32u4 FONA, (vi) Feather M0 Wi-Fi, and (vii) Bluefruit LE Micro. 


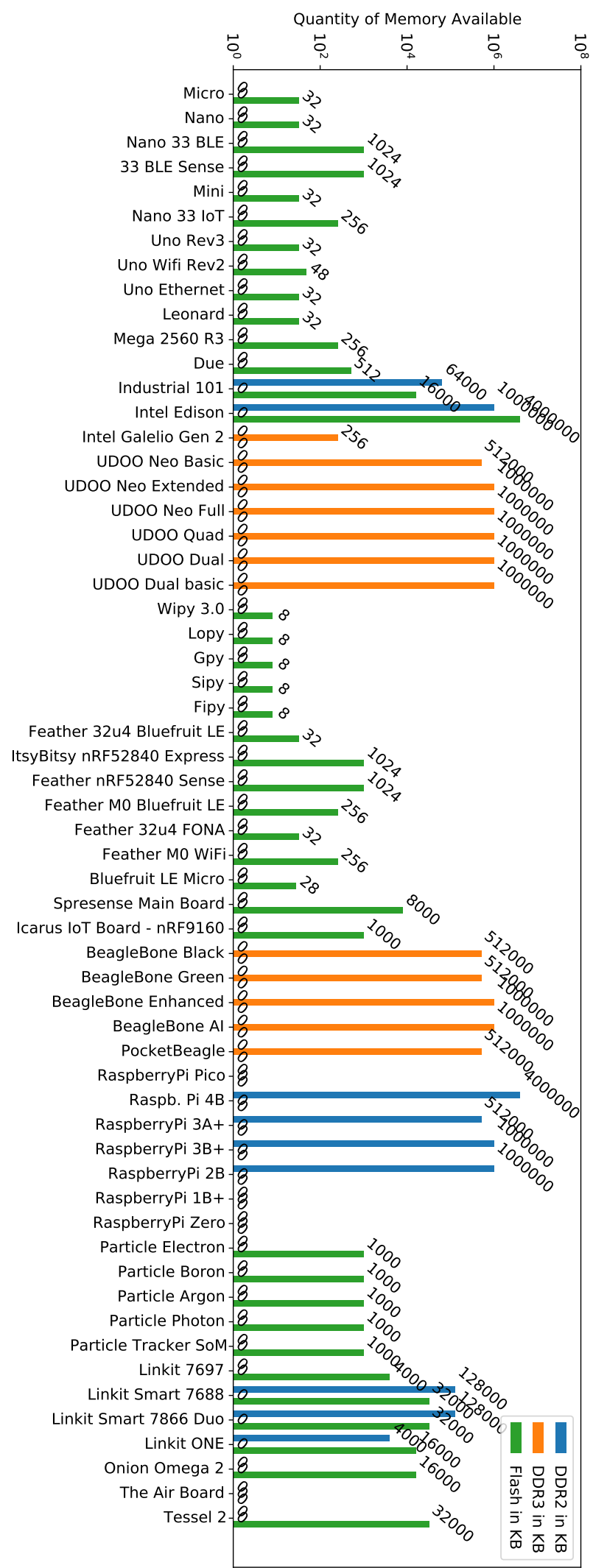

Fig. 8: Distribution of memory of each of the IoT computing boards for a combination of DDR2, DDR3, and Flash memories.

4) UDOO: The UDOO board constitutes an open-source manufacturer of mini PC compatible with Microsoft Windows, Android, and Linux distributions. The ODOO boards come under high computation power boards in the IoT domain. The boards considered from the UDOO family are as follows:

(i) UDOO Neo Basic, (ii) UDOO Neo Extended, (iii) UDOO Neo Full, (iv) UDOO Quad, (v) UDOO Dual, and (vi) UDOO Dual Basic.

5) Pycom: Platform Pycom provides end-to-end support for deploying IoT ideas. Pycom provides the hardware boards necessary for fast prototyping, associated software packages and imbibes various networking options starting from WiFi capable boards, Bluetooth, LoRa, SigFox, and cellular support for specific regions of interest. Pycom has its hardware proprietary and software open-sourced with micro-python as its preferred language of development with GPL v3. Moreover, it has different proprietary shields to perform various add-ons for each networking board. The boards considered from the Pycom family are as follows:

(i) Wipy3.0, (ii) Lopy, (iii) Gpy, (iv) Sipy, and (v) Fipy.

6) Particle: Particle is an entirely open-source hardware/software platform enabled with a cloud platform for implementing applications. The cloud platform provides a way to deploy applications. The boards also contain connectivity systems that support SIM cards and provide data plans. The communication protocols are configurable. The Particleboards have a specific OS that can be utilized for building suitable applications. The boards considered from the Particle family are as follows:

(i) Particle Electron, (ii) Particle Boron, (iii) Particle Photon, and (iv) Particle Tracker SoM.

7) BeagleBoard.org along with SanCloud and SeeedStudio: BeagleBone is an open-source project of both hardware and software. The boards are capable of extensive computation. Operating systems of Debian Linux and Android are supported. The following set of computing boards is of high clock speed, reflecting the instruction execution speed at the IoT node. The boards considered from the BeagleBone family are as follows:

(i) BeagleBone Black, (ii) BeagleBone Green, (iii) BeagleBone Enhanced, (iv) BeagleBone AI, and (v) PocketBeagle.

8) MediaTek: An IoT segment of MediaTek boards consists of Linkit series of boards belonging to the high-speed clock, replicating high computing speed capability. The MediaTek Linkit Series provides compatibility with Arduino IDE and Blockyduino IDE. Also, Linkit series boards are configurable using LinkIt SDK. The MediaTek IoT segment also provides a cloud-based platform to build and deploy IoT-oriented applications. The boards considered from the MediaTek family are as follows:

(i) LinkIt 7697, (ii) LinkIt Smart 7688, (iii) LinkIt Smart 7688 Duo, and (iv) LinkIt ONE.

9) Other sets of boards: Besides the boards mentioned above from different companies, several boards stand unique in their specified production. In the following, vendors are enlisted along with the respective boards and their support community or vendor.

(i) Intel Edison: Intel company and Arduino, (ii) Intel Galileo Gen 2: Intel company and Arduino, (iii) Spresense main board: Sony company, (iv) Icarus IoT board: Actinius company, (v) Onion Omega 2: Onion company, (vi) The Air 
board: The Air Things company, and (vii) Tessel 2: Tessel company.

\section{E. Network Interface}

Internet interface relates to the method of networking adopted, including Wi-Fi, Bluetooth, BLE, Ethernet, or in certain specific boards, we can see cellular network being used along with a SIM-card (eSIM, MicroSIM, and SIM card). WiFi provision is also demarcated into two levels (i) board with access to Wi-Fi and (ii) board with Wi-Fi and SoftAP. SoftAP configures the board to enable the functions of a Wi-Fi access point.

The boards with cellular connectivity are listed without mentioning their specificity in Table II. The inclusion of cellular network interface into IoT boards makes them capable of using a range of cellular network technologies including, $2 \mathrm{G}, 3 \mathrm{G}, 4 \mathrm{G}$, and 5G, which can serve many applications that from Short Messaging Service (SMS), Narrow Band IoT (NBIoT) communications, and even multi-media communications.

NB-IoT is a part of the Low Power Wide Area (LPWA) technology that significantly decreases the power consumption of user devices, enhances system capacity, and efficiently utilizes the spectrum, especially useful in deep coverage applications.

\section{TABLE II}

Cellular Technology-enabled Computing Boards

\begin{tabular}{|l|c|}
\hline Name of the Boards & Availability of Cellular Technology \\
\hline Pycom Gpy [52] & LTE CAT M1-NB \\
Pycom Fipy [49] & LTE CAT M1-NB1 \\
Adafruit Feather 32u4 FONA 82$]$ & GSM800 band Support \\
Icarious Board 55 & LTE Support \\
\hline
\end{tabular}

The boards with SigFox connectivity are listed in Table III. Low power protocols such as SigFox modules are the best choice for IoT devices though they have a certain set of challenges in their operational abilities. SigFox modules are known for their low implementation costs and low power consumption [86].

TABLE III

SigFox-enabled Computing Boards

\begin{tabular}{|l|c|}
\hline Name of the Boards & Availability of SigFox Technology \\
\hline Pycom Fipy [49] & Yes \\
Pycom Sipy [53] & Yes \\
The Air Board $[84]$ & Yes \\
\hline
\end{tabular}

The boards with LoRa connectivity are listed in Table IV LoRa modules are also known for their wide-range and lowpower networking capabilities and are widely used in various IoT applications [87]. The presence of such a facility is also analyzed in the boards considered.

A separate table has been provided to understand the availability of Ethernet, Wi-Fi, SoftAP, and BLE for each board. These technologies are most widely adopted due to easy access to compatible hardware. Table $\mathrm{V}$ provides information on the availability of the technologies mentioned above at each of the boards. A developer can select suitable combinations of
TABLE IV

LoRa-enabled Computing Boards

\begin{tabular}{|l|c|}
\hline Name of the Boards & Availability of LoRa Technology \\
\hline Pycom Fipy [49] & Yes \\
Pycom Lopy [51] & Yes \\
The Air Board [84] & Yes \\
\hline
\end{tabular}

network interfaces required for a particular application using this information.

\section{OBSERVATIONS AND DISCUSSION}

The paper compares 60 boards based on the parameters listed in Section III Most of the boards are grouped and categorized based on their origin and a suitable diversity in the analysis. Each of the parameters gives a numeric understanding of the boards. The numerical insights study the architectural construct of the considered IoT boards.

In this section, a recap analysis is done to understand the outcomes of a comparative study done in Section IV. That will be followed by some exceptional features of specific boards that inspire future board manufacturers. Some generic quantitative results that are obtained from the above comparative analysis are as follows:

- The range of pins on boards varies from 18 to 90, while the number of analog pins (i.e., DAC) ranges from 0 to 18 , the digital pins lie in the range of 10 to 78 . The total number of pins shows the range of devices attached to an IoT board. The pertinent ratio of analog-to-digital pins is $25 \%$. This finding can be correlated to the requirement of such a division. The analog requirement has been minimal in many applications using digital processing units.

- The mean of analog pins counts to 7.20, and that of digital pins counts to 30.80 , and the mean of a total number of available pins is computed as 38. The number of available pins in an average sense directs to the application capability of the boards at hand that we have used for comparison.

- The clock clusters include 12 very high-speed clocks $(>=800 \mathrm{MHz}), 19$ high-speed clock boards, and 30 low-speed clocks $(<192 \mathrm{MHz})$ for various applications purposes.

- The distribution of memory is considered for all the 60 IoT boards, and the following points need to be noted:

1) The number of boards that include DRAM (inclusive of other memory systems) counts to 6 , and the mean value of DRAM available at the compute board is around $278 \mathrm{MB}$.

2) The number of boards that include SRAM (inclusive of other memory systems) is 34 , and the mean value of SRAM available at the compute board is around $211 \mathrm{~KB}$.

3) The number of boards that include EEPROM (inclusive of other memory systems) is 11 , and the mean value of EEPROM available at the compute board is around $2 \mathrm{~KB}$. The number of boards that include 
TABLE V

Availability of Network Interface

\begin{tabular}{|c|c|c|c|c|}
\hline Name of the Boards & Ethernet & Wi-F & SoftAF & Bluetooth/BLE \\
\hline Micro [73] & No & No & No & No \\
\hline Nano |74| & No & No & No & No \\
\hline Nano 33 BLE 66 & No & No & Yes & Yes \\
\hline Nano 33 IoT 68$]$ & No & Yes & No & No \\
\hline Uno Rev3 |76| & No & No & No & No \\
\hline Uno Wi-Fi Rev2 |77| & No & Yes & No & No \\
\hline Uno Ethernet $\mid 78$ & Yes & No & No & No \\
\hline Leonard |79| & No & No & No & No \\
\hline Mega 2560 R3 [80 & No & No & No & No \\
\hline Due 60 & No & No & No & No \\
\hline Industrial 101 |47| & Yes & Yes & No & No \\
\hline Intel Edison |46| & Yes & Yes & No & No \\
\hline Intel Galileo Gen 2 |72| & Yes & Yes & No & No \\
\hline UDOO Neo Basic $|40|$ & Yes & No & Yes & Yes \\
\hline UDOO Neo Extended |40| & No & Yes & Yes & Yes \\
\hline UDOO Neo Full [40] & Yes & Yes & Yes & Yes \\
\hline UDOO Quad |41| & Yes & Yes & Yes & Yes \\
\hline UDOO Dual $\mid 41$ & Yes & Yes & Yes & Yes \\
\hline UDOO Dual Basic 41$]$ & Yes & Yes & Yes & Yes \\
\hline Wipy3.0 [50] & No & Yes & Yes & Yes \\
\hline Lopy $\mid 51$ & No & Yes & Yes & Yes \\
\hline Gpy |52] & No & Yes & Yes & Yes \\
\hline Sipy $\mid 53$ & No & Yes & Yes & Yes \\
\hline Fipy | 49 & No & Yes & Yes & Yes \\
\hline Feather 32u4 Bluefruit LE 81] & No & No & Yes & Yes \\
\hline ItsyBitsy nRF52840 Express [64] & No & No & Yes & Yes \\
\hline Feather nRF52840 Sense 65 & No & No & Yes & Yes \\
\hline Feather M0 Bluefruit LE $[\overline{69}]$ & No & No & Yes & Yes \\
\hline Feather 32u4 FONA |82] & No & Yes & Yes & Yes \\
\hline Bluefruit LE Micro [83] & No & No & Yes & Yes \\
\hline Spresence Main Board $\mid 56$ & No & No & No & No \\
\hline Icarus IoT Board - nRF9160 |55| & No & No & No & No \\
\hline BeagleBone Black 34 & Yes & No & No & No \\
\hline BeagleBone Green $|\overline{35}|$ & Yes & No & No & No \\
\hline BeagleBone Enhanced $|36|$ & Yes & Yes & Yes & Yes \\
\hline BeagleBone AI 31$]$ & Yes & Yes & Yes & Yes \\
\hline PocketBeagle |37| & No & No & No & No \\
\hline Raspberry Pi Pico [57 & No & No & No & No \\
\hline Raspberry Pi 4 B |30| & Yes & Yes & Yes & Yes \\
\hline Raspberry Pi 3 A+ 32 & Yes & Yes & Yes & Yes \\
\hline Raspberry Pi $3 \mathrm{~B}+[\overline{33}]$ & Yes & Yes & Yes & Yes \\
\hline Raspberry Pi 2 B [39] & Yes & No & No & No \\
\hline Raspberry Pi 1 B+ [42] & Yes & No & No & No \\
\hline Raspberry Pi Zero [38| & No & Yes & Yes & Yes \\
\hline Particle Electron [59| & No & No & No & No \\
\hline Particle Boron 61] & No & No & Yes & Yes \\
\hline Particle Argon [62] & No & No & Yes & Yes \\
\hline Particle Photon $|\overrightarrow{58}|$ & No & Yes & No & No \\
\hline Particle Tracker SoM 63] & No & Yes & Yes & Yes \\
\hline Linkit 7697 [54] & No & Yes & Yes & Yes \\
\hline Linkit Smart 7688 |44| & Yes & Yes & No & No \\
\hline Linkit Smart 7866 Duo 44 & Yes & Yes & No & No \\
\hline Linkit ONE 48 & No & No & Yes & Yes \\
\hline Onion Omega 2 [43] & Yes & Yes & No & No \\
\hline The Air Board [84] & No & Yes & Yes & Yes \\
\hline Tessel 2 |71] & Yes & Yes & No & No \\
\hline
\end{tabular}

Flash (inclusive of other memory systems) is 39 , and the mean value of Flash memory available at the compute board is around $107 \mathrm{MB}$.

4) The number of boards that include DDR2 (inclusive of other memory systems) is 10 , and the mean value of DDR2 available at the compute board is around $863 \mathrm{MB}$.

5) The number of boards that include DDR3 (inclusive of other memory systems) is 12 , and the mean value of DDR3 available at the compute board is around $736 \mathrm{MB}$.

The values mentioned earlier for pin allocations, memory division, and clock speed availability of the selected IoT computing boards launched during the years 2008-2021. However, the analysis provides us with a numeric understanding of the future requirements of the upcoming IoT boards. Conversely, a qualitative understanding of a specific set of IoT boards helps us understand the requirements of potential future IoT computing boards. A few such vital insights are listed in the following.

- Presence of SD Card slots (including other memory systems) for the selected IoT boards depending on the application scenarios.

- Availability of various levels of support for developing the IoT products and fast prototyping. Examples of such support levels are as follows:

1) Support from the software developer communities for various library development is required to interface various hardware modules. For example, IoT boards need extensive hardware libraries. A software group generally develops the required libraries in the academic communities or industries to support various applications.

2) On the other hand, the hardware developer communities develop different versions of similar IoT boards (e.g., Arduino support and Adafruit developers). Such developments are essential for target applications.

3) IDE platform is another support provided from the vendors for testing and prototyping IoT boards faster (e.g., Arduino, Pycom, Particle, Tessel, and Onion are a few to mention from the chosen boards). In particular, IDE platforms help users rapidly execute, debug, and deploy programming modules on such IoT boards and, thus, enable them for faster prototyping.

4) Software/OS flexibility provision for usage in multiple work environments (such as Raspberry $\mathrm{Pi}$, Beagle boards, and Intel boards) is also highly crucial.

5) Cloud provision for deployment and maintenance is also one of the essential enablers for various applications (e.g., Particle and MediaTek companies provide integrated Cloud IoT systems). In particular, IoT integrated cloud platforms are now available to enable direct cloud access to the computing stations/nodes for data processing and actuation.

6) GPS/GPRS/GNSS support for geo-locating capabilities is very useful for automotive IoT applications. A few boards are Adafruit Feather 32u4 FONA [82], Spressence main baord [56], Icarus IoT board [55], and the Air board extensions [84]). 
- Ease of accessing the existing and evolved connectivity options for the future IoT boards. In particular, the future IoT boards should perform under low power, low data rate, and support long-ranged communications to integrate with the existing and next-generation cellular technologies. For example, the importance of NB-IoT, SigFox, and LoRa lies in their ability to perform under low power, low data rate, and support long-range communications. Moreover, some conditions apply to IoT sensor boards deployed for monitoring systems.

- Compatibility with multiple access protocols to connect to the other devices. In particular, from our selected 60 IoT computing boards 24 boards are provided with the Ethernet interface, 34 boards with essential Wi-Fi access provision, 5 boards are provided with Wi-Fi and SoftAPs, and 32 boards are provided with Bluetooth/BLE capability. In addition, the new forms of communication protocols are to be included in the architectural design of the future IoT boards to widen the application domain.

\section{CONCLUSION}

This survey quantitatively studied selected 60 IoT computing boards based on their different capabilities, such as clock speed, memory, and network interfaces. In particular, the processor used in each IoT board is identified to assess the processing power available at the board. For example, Raspberry Pi 4B and Beagle Bone AI were identified with a tremendous clock-speed and capable of executing the AIbased applications. We also noticed that the accelerated GPU blocks for image processing and VR applications are essential. Moreover, the availability of multiple channels to perform the networking applications were studied. In addition, the development of application-specific boards with multiple cores and GPU-enabled acceleration, availability of wide networking technology, and presence of small form-factor identified as a set of niche technological advancements necessary for the upcoming boards. Further, also discussed the value of a reach community support for an end-to-end product development and rapid prototyping when present and upcoming IoT boards are considered. In particular, this comparative survey has brought out the advancements in the growing IoT industries and highlighted the possible direction of IoT board manufacturing based on the future application scenarios.

\section{REFERENCES}

[1] D. R. Roberto Minerva, Abyi Biru, Towards a Definition of the Internet of Things (IoT), May 2015.

[2] J. Gubbi, R. Buyya, S. Marusic, and M. Palaniswami, "Internet of things (IoT): A vision, architectural elements, and future directions," Future Generation Computer Systems, vol. 29, no. 7, pp. 1645-1660, 2013. [Online]. Available: https://www.sciencedirect.com/science/article/pii/ S0167739X13000241

[3] K. L. Bober, V. Jungnickel, M. Emmelmann, M. Riegel, E. Tangdiongga, A. M. J. Koonen, J. . P. Linnartz, D. Behnke, M. Müller, P. . B. Bök, S. V. Colonques, and M. M. Vazquez, "A flexible system concept for $\mathrm{Li}-\mathrm{Fi}$ in the internet of things," in 2020 22nd International Conference on Transparent Optical Networks (ICTON), 2020, pp. 1-4.

[4] J. Tang, D. Sun, S. Liu, and J. Gaudiot, "Enabling deep learning on IoT devices," Computer, vol. 50, no. 10, pp. 92-96, 2017.
[5] N. Neshenko, E. Bou-Harb, J. Crichigno, G. Kaddoum, and N. Ghani, "Demystifying IoT security: An exhaustive survey on IoT vulnerabilities and a first empirical look on internet-scale IoT exploitations," IEEE Communications Surveys Tutorials, vol. 21, no. 3, pp. 2702-2733, 2019.

[6] M. A. López Peña and I. Muñoz Fernández, "SAT-IoT: An architectural model for a high-performance fog/edge/cloud IoT platform," in 2019 IEEE 5th World Forum on Internet of Things (WF-IoT), 2019, pp. 633638.

[7] P. Marwedel, Embedded System Design, Embedded Systems Foundations of Cyber-Physical Systems, and the Internet of Things. TU Dortmund, Dortmund, Germany: Springer, 2021.

[8] Road vehicles - Controller area network (CAN), ISO Std. 11898-1, 2015.

[9] D. S. Dawoud and P. Dawoud, Serial Communication Protocols and Standards RS232/485, UART/USART, SPI, USB, INSTEON, Wi-Fi and WiMAX. IEEE, 2020.

[10] IEEE, "IEEE standard for high-speed test access port and on-chip distribution architecture," IEEE Std 1149.10-2017, pp. 1-96, 2017.

[11] J. Saadé, F. Pétrot, A. Picco, J. Huloux, and A. Goulahsen, "A systemlevel overview and comparison of three high-speed serial links: USB 3.0, PCI Express 2.0 and LLI 1.0," in 2013 IEEE 16th International Symposium on Design and Diagnostics of Electronic Circuits Systems (DDECS), 2013, pp. 147-152.

[12] P. Ray, "A survey on internet of things architectures," Journal of King Saud University - Computer and Information Sciences, vol. 30, no. 3, pp. 291-319, 2018. [Online]. Available: https://www.sciencedirect.com/ science/article/pii/S1319157816300799

[13] A. R. Chandan and V. D. Khairnar, "Bluetooth low energy (BLE) crackdown using IoT," in 2018 International Conference on Inventive Research in Computing Applications (ICIRCA), 2018, pp. 1436-1441.

[14] R. P. Jover and I. Murynets, "Connection-less communication of IoT devices over LTE mobile networks," in 2015 12th Annual IEEE International Conference on Sensing, Communication, and Networking (SECON), 2015, pp. 247-255.

[15] A. Lavric, A. I. Petrariu, and V. Popa, "SigFox communication protocol: The new era of IoT?" in 2019 International Conference on Sensing and Instrumentation in IoT Era (ISSI), 2019, pp. 1-4.

[16] J. Garcia, M. March, L. Cerda, J. Corbal, and M. Valero, "On the design of hybrid DRAM/SRAM memory schemes for fast packet buffers," in 2004 Workshop on High Performance Switching and Routing, 2004. HPSR., 2004, pp. 15-19.

[17] K. V. Nikiforov, Y. Chaplygin, V. V. Losev, and S. S. Kartashev, "EEPROM memory block for use in a micro-power RFID tag," in 2018 IEEE Conference of Russian Young Researchers in Electrical and Electronic Engineering (EIConRus), 2018, pp. 2007-2010.

[18] C. Shuang-yan, W. Dong-hui, S. Rui, and H. Chao-huan, "An innovative design of the DDR/DDR2 SDRAM compatible controller," in 2005 6th International Conference on ASIC, vol. 1, 2005, pp. 62-66.

[19] X. Wang, J. Wang, X. Tao, M. Yang, and J. Lai, "Design and implementation of DDR3 SDRAM controller," in 2018 14th IEEE International Conference on Solid-State and Integrated Circuit Technology (ICSICT), 2018, pp. 1-3.

[20] J. Zheng, K. Yan, Y. Zhang, and Z. Chen, "Design and implementation of DDR4 SDRAM controller based on FPGA," in 2018 2nd IEEE Advanced Information Management,Communicates,Electronic and Automation Control Conference (IMCEC), 2018, pp. 421-424.

[21] Jeremy Carter and Eric Grommonb and Phil Harris, "From conceptual to operational: Over-the-air-programming of land mobile radios," Physical Communication, no. 19, 2016.

[22] H. B. Yesilyurt, H. Aksu, S. Uluagac, and R. Beyah, "SOTA: Secure over-the-air programming of IoT devices," in MILCOM 2018 - 2018 IEEE Military Communications Conference (MILCOM), 2018, pp. 1-8.

[23] N. Sornin, "LoRaWAN®:firmware updates Over-the-Air," in Semtech Technical Paper, 200 Flynn Road Camarillo, CA 93012-8790 USA, 2020.

[24] Official U.S. government information about the Navstar Global Positioning System (GPS) and related topics, 2021, (Accessed on 25/12/2021). [Online]. Available: https://www.gps.gov/technical/icwg/

[25] S. Zaidman, "GLOBAL POSITIONING SYSTEM WIDE AREA AUGMENTATION SYSTEM (WAAS) PERFORMANCE STANDARD," Department of Transportation USA, Federal Aviation Administration, DOT is at 1200 New Jersey Ave SE, Washington, DC 20590, United States and FAA is at 800 Independence Ave., S.W., Tech. Rep., 2008.

[26] P. Rakshit, S. Dan, B. Das, A. Santra, S. Mahato, and A. Bose, "Compact, low-cost, single-frequency NavIC receiver development," in 2020 URSI Regional Conference on Radio Science (URSI-RCRS), 2020, pp. $1-4$ 
[27] Jianping Yuan, Huamin Jia, and Qun Fang, "Application of GPS to space vehicles: analysis of space environment and errors," IEEE Aerospace and Electronic Systems Magazine, vol. 13, no. 1, pp. 25-30, 1998.

[28] A. Garrigós, D. Marroquí, J. M. Blanes, R. Gutiérrez, I. Blanquer, and M. Cantó, "Designing arduino electronic shields: Experiences from secondary and university courses," in 2017 IEEE Global Engineering Education Conference (EDUCON), 2017, pp. 934-937.

[29] A. Nayyar and V. Puri, "A review of arduino board's, lilypad's arduino shields," in 2016 3rd International Conference on Computing for Sustainable Global Development (INDIACom), 2016, pp. 1485-1492.

[30] Raspberry Pi 4B, Raspberry Pi, 2021, (Accessed on 26/11/2021). [Online]. Available: https://datasheets.raspberrypi.org/rpi4/ raspberry-pi-4-product-brief.pdf

[31] BeagleBone AI Board, BeagleBone.org, 2019, (Accessed on 26/11/2021). [Online]. Available: https://beagleboard.org/ai

[32] Raspberry Pi 3A+, Raspberry Pi, 2018, (Accessed on 26/11/2021). [Online]. Available: https://datasheets.raspberrypi.org/rpi3/ raspberry-pi-3-aplus-product-brief.pdf

[33] Raspberry Pi 3B+, Raspberry Pi, 2018, (Accessed on 26/11/2021). [Online]. Available: https://static.raspberrypi.org/files/product-briefs/ 200206+Raspberry+Pi+3+Model+B+plus+Product+Brief+PRINT\& DIGITAL.pdf

[34] BeagleBone Black Development Board, BeagleBone.org, 2013, (Accessed on 26/11/2021). [Online]. Available: https://beagleboard.org/ black

[35] BeagleBone Green Development Board, SeeedStudio, 2019, (Accessed on 26/11/2021). [Online]. Available: https://beagleboard.org/green

[36] BeagleBone Enhanced Development Board, SanCloud, 2019, (Accessed on 26/11/2021). [Online]. Available: https://beagleboard.org/enhanced

[37] PocketBeagle, BeagleBone.org, 2008, (Accessed on 26/11/2021). [Online]. Available: https://beagleboard.org/pocket

[38] Raspberry Pi Zero, Raspberry Pi, 2012, (Accessed on 26/11/2021). [Online]. Available: https://cdn.sparkfun.com/assets/learn_tutorials/6/7/ 6/PiZero_1.pdf

[39] Raspberry Pi 2B, Raspberry Pi, 2012, (Accessed on 26/11/2021). [Online]. Available: https://cdn-shop.adafruit.com/pdfs/raspberrypi2modelb. pdf

[40] Discover the UDOO NEO: Your Personal IoT Maker Board, Mar. 2021. [Online]. Available: https://www.udoo.org/udoo-neo/

[41] Discover the UDOO QUAD/DUAL: Android Linux Arduino singleboard PC, Sep. 2020. [Online]. Available: https://www.udoo.org/ udoo-quad-dual/

[42] Raspberry Pi 1B+, Raspberry Pi, 2012, (Accessed on 26/11/2021). [Online]. Available: https://cdn-shop.adafruit.com/datasheets/pi-specs. pdf

[43] Onion Omega - 2 Data Sheet, Onion Corporation, Boston, Toronto and Shenzhen.

[44] MediaTek Linkit 7688, MediaTek, 2016, (Accessed on 26/11/2021). [Online]. Available: https://labs.mediatek.com/en/platform/ linkit-smart-7688

[45] Intel Edison Compute Module Data Sheet, Intel - Arduino, 2200 Mission College Blvd.,Santa Clara, CA 95054-1549, USA.

[46] IntelEdison. [Online]. Available: https://www.arduino.cc/en/ ArduinoCertified/IntelEdison

[47] Arduino Industrial 101, (Accessed on 26/11/2021). [Online]. Available: https://www.sparkfun.com/products/retired/14134

[48] MediaTek Linkit ONE, MediaTek, 2016, (Accessed on 26/11/2021). [Online]. Available: https://labs.mediatek.com/en/platform/linkit-one

[49] Fipy 1.0, Pycom, 2017, version 1.0, (Accessed on 25/12/2021) [Online]. Available: https://pycom.io/wp-content/uploads/2017/08/ fipySpecsheetAugust2017n2-1.pdf

[50] Wipy 3.0, Pycom, 2020, version 1.0,(Accessed on 25/12/2021). [Online]. Available: https://pycom.io/wp-content/uploads/2018/08/ Pycom_Specsheet_WiPy3.0.pdf

[51] Lopy, Pycom, 2017, version 1.0,(Accessed on 25/12/2021). [Online] Available: https://pycom.io/wp-content/uploads/2018/08/lopy-specsheet. pdf

[52] Gpy, Pycom, 2017, version 1.0, (Accessed on 25/12/2021). [Online]. Available: https://pycom.io/wp-content/uploads/2018/08/gpy-specsheet. pdf

[53] Sipy, Pycom, 2017, version 1.0, (Accessed on 25/12/2021). [Online] Available: https://pycom.io/wp-content/uploads/2018/08/sipy-specsheet. pdf

[54] MediaTek Linkit 7697, MediaTek, 2016, (Accessed on 26/11/2021). [Online]. Available: https://labs.mediatek.com/en/chipset/MT7697
[55] Icarus IoT Development Board, Actinius, 2019, (Accessed on 26/11/2021). [Online]. Available: https://docs.actinius.com/icarus/ datasheet\#overview

[56] Sony Spresense Main Board, Sony, 2018, (Accessed on 26/11/2021). [Online]. Available: https://developer.sony.com/develop/spresense/docs/ introduction_en.html\#_spresense_hardware

[57] Raspberry Pi Pico, Raspberry Pi, 2012, (Accessed on 26/11/2021). [Online]. Available: https://datasheets.raspberrypi.org/pico/pico-datasheet. pdf

[58] Particle Photon IoT development board, Particle, 2020, (Accessed on 26/11/2021). [Online]. Available: https://docs.particle.io/assets/pdfs/ datasheets/photon-datasheet.pdf

[59] Particle Electron, Particle, 2020, (Accessed on 26/11/2021). [Online]. Available: https://docs.particle.io/datasheets/electron/electron-datasheet/ \#functional-description

[60] Arduino Due, (Accessed on 26/11/2021). [Online]. Available: https: //store.arduino.cc/usa/due

[61] Particle Boron, Particle, 2020, (Accessed on 26/11/2021). [Online]. Available: https://docs.particle.io/assets/pdfs/datasheets/boron-datasheet. pdf

[62] Particle Argon IoT development board, Particle, 2020, (Accessed on 26/11/2021). [Online]. Available: https://docs.particle.io/assets/pdfs/ datasheets/argon-datasheet.pdf

[63] Particle Tracker 1G/2G/3G, Particle, 2020, (Accessed on 26/11/2021). [Online]. Available: https://docs.particle.io/assets/pdfs/ datasheets/tracker-som-datasheet.pdf

[64] Adafruit ItsyBitsy nRF52840 Express, Adafruit Learning System, 2020, (Accessed on 26/11/2021). [Online]. Available: https://cdn-learn.adafruit.com/downloads/pdf/ adafruit-itsybitsy-nrf52840-express.pdf?timestamp $=1580862368$

[65] K. Rembor, Adafruit Feather nRF52840 Sense, Adafruit Learning System, 2020, (Accessed on 26/11/2021). [Online]. Available: https://cdn-learn.adafruit.com/downloads/pdf/ adafruit-feather-sense.pdf?timestamp $=1619064343$

[66] Arduino Nano 33 BLE, (Accessed on 26/11/2021). [Online]. Available: https://store.arduino.cc/usa/nano-33-ble

[67] Arduino Nano 33 BLE Sense, (Accessed on 26/11/2021). [Online]. Available: https://store.arduino.cc/usa/nano-33-\{BLE\}-sense

[68] Arduino Nano 33 IoT, (Accessed on 26/11/2021). [Online]. Available: https://store.arduino.cc/usa/nano-33-IoT

[69] L. Ada, Adafruit Feather MO Bluefruit LE, Adafruit Learning System, 2016, (Accessed on 26/11/2021). [Online]. Available: https://cdn-learn. adafruit.com/downloads/pdf/adafruit-feather-m0-bluefruit-le.pdf

[70] Adafruit Feather MO WiFi with ATWINC1500, (Accessed on 26/11/2021). [Online]. Available: https://learn.adafruit.com/ adafruit-feather-m0-wifi-atwinc 1500

[71] Onion Omega - 2 Data Sheet, Onion Corporation, Boston, Toronto and Shenzhen.

[72] IntelGalileo. [Online]. Available: https://www.arduino.cc/en/ ArduinoCertified/IntelGalileo

[73] Arduino Micro, (Accessed on 26/11/2021). [Online]. Available: https: //store.arduino.cc/usa/arduino-micro

[74] Arduino Nano, (Accessed on 26/11/2021). [Online]. Available: https: //store.arduino.cc/usa/arduino-nano

[75] T. A. Team, Getting Started with the Arduino Mini, (Accessed on 26/11/2021). [Online]. Available: https://www.arduino.cc/en/Guide/ ArduinoMini

[76] Arduino Uno Rev3, (Accessed on 26/11/2021). [Online]. Available: https://store.arduino.cc/usa/arduino-uno-rev3

[77] ARDUINO UNO WiFi REV2, (Accessed on 26/11/2021). [Online]. Available: https://store.arduino.cc/usa/arduino-uno-wifi-rev2

[78] ArduinoBoardEthernet, (Accessed on 26/11/2021). [Online]. Available: https://www.arduino.cc/en/Main/ArduinoBoardEthernet/

[79] Arduino Leonardo with Headers, (Accessed on 26/11/2021). [Online]. Available: https://store.arduino.cc/usa/leonardo

[80] Arduino Mega 2560 Rev3, (Accessed on 26/11/2021). [Online]. Available: https://store.arduino.cc/usa/mega-2560-r3

[81] L. Ada, Adafruit Feather $32 u 4$ Bluefruit LE, Adafruit Learning System, 2015. [Online]. Available: https://cdn-learn.adafruit.com/downloads/pdf/ adafruit-feather-32u4-bluefruit-le.pdf

[82] Adafruit Feather 32u4 FONA, Adafruit Learning System, 2016, (Accessed on 26/11/2021). [Online]. Available: https://cdn-learn. adafruit.com/downloads/pdf/adafruit-feather-32u4-fona.pdf

[83] L. Ada, Adafruit Bluefruit LE micro, Adafruit Learning System, 2016, (Accessed on 26/11/2021). [Online]. Available: $\quad$ https://cdn-learn.adafruit.com/downloads/pdf/ 
bluefruit-le-micro-atmega32u4-microcontroller-usb-bluetooth-le-in-one pdf

[84] Airboard - AirThings Inc., 2015, (Accessed on 26/11/2021). [Online]. Available: http://www.theairboard.cc/tech-specs/

[85] S. Abinesh, M. Kathiresh, and R. Neelavenik, "Analysis of multicore architecture for automotive applications," in 2014 International Conference on Embedded Systems (ICES), 2014, pp. 76-79.

[86] A. Lavric, A. I. Petrariu, and V. Popa, "Long range SigFox communication protocol scalability analysis under large-scale, high-density conditions," IEEE Access, vol. 7, pp. 35 816-35 825, 2019.

[87] U. Noreen, A. Bounceur, and L. Clavier, "A study of LoRa low power and wide area network technology," in 2017 International Conference on Advanced Technologies for Signal and Image Processing (ATSIP), 2017, pp. 1-6. 